\title{
A NECESSARY AND SUFFICIENT CONDITION FOR CONVERGENCE IN LAW OF RANDOM SUMS OF RANDOM VARIABLES UNDER NONRANDOM CENTERING
}

\author{
MARK FINKELSTEIN AND HOWARD G. TUCKER
}

(Communicated by William D. Sudderth)

\begin{abstract}
Let $\left\{X_{n}\right\}$ be a sequence of independent, identically distributed (i.i.d.) random variables with common mean $\mu \neq 0$ and variance $\sigma^{2}>0$. Let $\left\{S_{n}\right\}$ be a sequence of nonnegative integer-valued random variables such that for each $n$ the random variables $S_{n}, X_{1}, X_{2}, \ldots$ are independent. Then $\left(X_{1}+\cdots+X_{S_{n}}-n \mu\right) / \sqrt{n \sigma^{2}} \stackrel{\mathscr{L}}{\longrightarrow}$ (some) $Z$ if and only if $\left(S_{n}-n\right) / \sqrt{n} \stackrel{\mathscr{L}}{\longrightarrow}$ (some) $U$, in which case the distribution of $Z$ is that of $X+Y$, where $X$ and $Y$ are independent random variables, $X$ being $\mathcal{N}(0,1)$ and $Y$ having the same distribution as $\mu U / \sigma$.
\end{abstract}

\section{INTRODUCTION AND SUMMARY}

A necessary and sufficient condition is obtained here for a random sum of independent random variables with nonrandom centering and norming to converge in law to a limit law. More precisely, let $\left\{X_{n}\right\}$ be a sequence of independent, identically distributed (i.i.d.) random variables with common mean $\mu \neq 0$ and variance $\sigma^{2}>0$. Let $\left\{S_{n}\right\}$ be a sequence of nonnegative integervalued random variables such that for each $n$, the random variables $S_{n}, X_{1}$, $X_{2}, \ldots$ are independent. Let us denote

$$
Z_{n}=\frac{X_{1}+\cdots+X_{S_{n}}-n \mu}{\sqrt{n \sigma^{2}}},
$$

which is an independent random sum of i.i.d. random variables with nonrandom centering. We shall use the notation $T_{n} \stackrel{\mathscr{L}}{\longrightarrow} T$ to mean that the distribution function of $T_{n}$ converges completely to that of $T$ as $n \rightarrow \infty$. We shall prove the following theorem.

Received by the editors January 21, 1989, and in revised form April 23, 1989. Presented at the 209th meeting of the Institute of Mathematical Statistics, at Davis, California, June 26, 1989.

1980 Mathematics Subject Classification (1985 Revision). Primary 60F05.

Key words and phrases. Random sums of random variables, nonrandom centering, convergence in law. 
Theorem. The condition

$$
Z_{n} \stackrel{\mathscr{L}}{\longrightarrow} \text { (some) } Z
$$

holds if and only if the condition

$$
\left(S_{n}-n\right) / \sqrt{n} \stackrel{\not L}{\longrightarrow} \text { (some) } U
$$

holds, in which case the distribution of $Z$ is that of $X+Y$, where $X$ and $Y$ are independent random variables, $X$ being $\mathcal{N}(0,1)$ and $Y$ having the same distribution as $\mu U / \sigma$.

An immediate consequence of this theorem is the following:

Corollary. A necessary and sufficient condition that $Z_{n} \stackrel{\mathscr{L}}{\longrightarrow} \mathscr{N}(0,1)$ is that

$$
\left(S_{n}-n\right) / \sqrt{n} \stackrel{P}{\longrightarrow} 0 .
$$

It should be noted that in our definition of $Z_{n}$ we are using fixed centering $n \mu$ rather than random centering $S_{n} \mu$. If random centering is used, then it is known and easily proved (see [4]) that if $S_{n} / n \stackrel{P}{\longrightarrow} 1$ as $n \rightarrow \infty$, then

$$
\frac{X_{1}+\cdots+X_{S_{n}}-S_{n} \mu}{\sqrt{n \sigma^{2}}} \stackrel{\mathscr{L}}{\longrightarrow} \mathcal{N}(0,1) \text {. }
$$

But this result does not necessarily hold if $S_{n} \mu$ is replaced by $n \mu$. For example, suppose each $X_{n}$ is $\mathscr{N}(-1,1)$ and suppose that $S_{n}=n \pm[\sqrt{n}]$ with probabilities $\frac{1}{2}, \frac{1}{2}$, where now and in the sequel $[x]$ means the largest integer $\leq x$. In this case the limit distribution is that of an equal mixture of $\mathscr{N}(-1,1)$ and $\mathscr{N}(1,1)$. The condition $\left(S_{n}-n\right) / \sqrt{n} \stackrel{\not}{\longrightarrow}$ (some) $U$ is stronger than $S_{n} / n \stackrel{P}{\longrightarrow} 1$ and is shown here to be necessary and sufficient for $Z_{n} \stackrel{\not}{\longrightarrow}$ (some) $Z$. As stated in the corollary, the even stronger condition $\left(S_{n}-n\right) / \sqrt{n} \stackrel{P}{\longrightarrow} 0$ is necessary and sufficient for $Z$ to be $\mathcal{N}(0,1)$.

Explicit mention of the following observations is in order. First, the result obtained here is not the first time that the question of nonrandom centering has been addressed. Z. Rychlik in [3] obtained one result giving sufficient conditions for convergence of random sums under nonrandom centering to a limit law. He assumed $E\left|S_{n}\right|<\infty$ for all $n$; his random variables $\left\{X_{n}\right\}$ are not identically distributed as are ours, but they do satisfy what he refers to as a "random Lindeberg condition." Second, examples are very easy to come by for $\left\{S_{n}\right\}$ to satisfy the basic hypothesis and the condition $\left(S_{n}-n\right) / \sqrt{n} \stackrel{\perp}{\longrightarrow}$ (some) $U$. Then J. G. Shanthikumar and U. Sumita in [5] proved sufficiency of this condition in a special case of our result. They assumed that each $S_{n}$ has a finite variance and that $S_{n} / n \stackrel{P}{\longrightarrow} \alpha, \operatorname{Var}\left(S_{n}\right) / n \rightarrow \beta^{2}$, and $\left(S_{n}-n \alpha\right) / n^{1 / 2} \beta \longrightarrow$ $\mathscr{N}(0,1)$. These assumptions were shown to imply that

$$
\left(X_{1}+\cdots+X_{S_{n}}-n \alpha \mu\right) /\left(n\left(\alpha \sigma^{2}+\beta^{2} \mu\right)\right)^{1 / 2} \stackrel{\leftrightarrow}{\longrightarrow}(0,1) .
$$

S. K. Basu, the reviewer of this paper in Mathematical Reviews, remarked that "this particular version of the central limit theorem for random sums of r.v.'s 
seems to be of greater utility from the point of view of applications" (see Mathematical Reviews 86d:60028). Thus further research in convergence in law of random sums under nonrandom centering appears to be justified.

\section{Proof of THE THEOREM}

The problem connected with nonrandom centering occurs only in the case $\mu \neq 0$. Without loss of generality we may assume that $\mu>0$. The following lemma will be used twice in the sequel:

Lemma 1. If $\left\{U_{n}\right\}$ is a sequence of random variables which is not tight, then there exists $\varepsilon>0$ and an increasing sequence of positive integers $\left\{k_{n}\right\}$ such that $P\left[U_{k_{n}} \geq n\right]>\varepsilon$ for all $n$ or $P\left[U_{k_{n}} \leq-n\right]>\varepsilon$ for all $n$.

Proof. If tightness is violated, then there exists an $\varepsilon>0$ such that for every $K>0, P\left[\left|U_{n}\right| \geq K\right]>2 \varepsilon$ for infinitely many values of $n$, from which it follows that, for all $K>0, P\left[U_{n} \geq K\right]>\varepsilon$ infinitely often or $P\left[U_{n} \leq-K\right]>\varepsilon$ infinitely often. Without loss of generality, assume that $P\left[U_{n} \geq K\right]>\varepsilon$ for every $K>0$ and for infinitely many values of $n$. For $K=1$ there exists a subsequence $\left\{k_{1, n}\right\}$ of the positive integers such that $k_{1, n} \uparrow \infty$ and such that $P\left[U_{k_{1, n}} \geq 1\right]>\varepsilon$ for all values of $n$. Select $k_{1}=k_{1,1}$. For $K=2$ there exists a subsequence $\left\{k_{2, n}\right\}$ of the positive integers such that $P\left[U_{k_{2, n}} \geq 2\right]>\varepsilon$ for all $n$. Select $k_{2}$ from $\left\{k_{2, n}\right\}$ such that $k_{2}>k_{1}$. Continuing in this manner we obtain $\left\{k_{n}\right\}$ such that $k_{n} \uparrow \infty$ and such that $P\left[U_{k_{n}} \geq n\right]>\varepsilon$.

Lemma 2. If either condition of the theorem holds, then the sequence $\left\{S_{n} / n\right\}$ is tight.

Proof. If $\left.\left\{S_{n}-n\right) / \sqrt{n}\right\}$ is tight, then for arbitrary $\varepsilon>0$ there exists $K_{\varepsilon}>0$ such that $P\left[\left|\left(S_{n}-n\right) / \sqrt{n}\right| \geq K_{\varepsilon}\right]<\varepsilon$ for all $n$. For all $n>\left(K_{\varepsilon} / \varepsilon\right)^{2}$, we have $\left.P\left[\mid S_{n} / n\right)-1 \mid \geq \varepsilon\right]<\varepsilon$, which implies that $S_{n} / n \stackrel{P}{\longrightarrow} 1$, which in turn implies that $\left\{S_{n} / n\right\}$ is tight. Next, suppose that

$$
Z_{n}=\frac{X_{1}+\cdots+X_{S_{n}}-n \mu}{\sqrt{n \sigma^{2}}} \stackrel{\mathscr{t}}{\longrightarrow} Z
$$

for some random variable $Z$. Let us assume to the contrary that $\left\{S_{n} / n\right\}$ is not tight. Since $P\left[S_{n} / n \geq 0\right]=1$ for all $n$, then by Lemma 1 there exists $\varepsilon>0$ and integers $k_{n} \uparrow \infty$ such that

$$
P\left[S_{k_{n}} \geq n k_{n}\right] \geq \varepsilon
$$


for all $n$. Let $\eta_{n}=(n-1) \sqrt{k_{n}} \mu / \sigma$; then $\eta_{n} \uparrow \infty$ as $n \rightarrow \infty$. Now, using independence of $S_{n}, X_{1}, X_{2}, \ldots$, we have

$$
\begin{aligned}
& P\left[Z_{k_{n}} \geq \eta_{n}\right] \geq P\left(\left[Z_{k_{n}} \geq \eta_{n}\right]\left[S_{k_{n}} \geq n k_{n}\right]\right)
\end{aligned}
$$

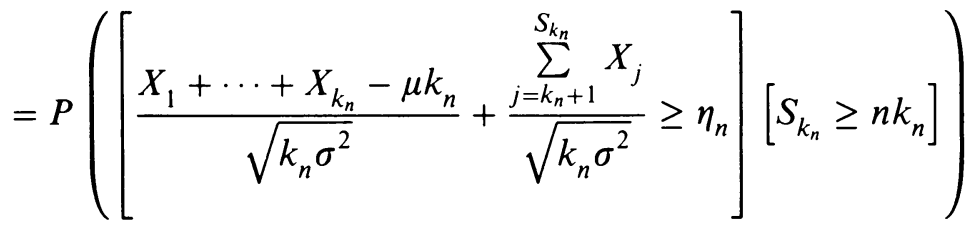

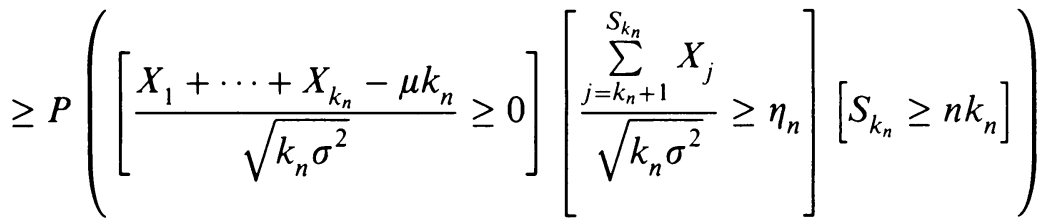

$$
\begin{aligned}
& =\sum_{m=n k_{n}}^{\infty} P\left[\frac{X_{1}+\cdots+X_{k_{n}}-\mu k_{n}}{\sqrt{k_{n} \sigma^{2}}} \geq 0\right] P\left[\frac{\sum_{j=k_{n}+1}^{m} X_{j}}{\sqrt{k_{n} \sigma^{2}}} \geq \eta_{n}\right] P\left[S_{k_{n}}=m\right] .
\end{aligned}
$$

Let $0<\delta<\frac{1}{2}$. By the Lindeberg-Lévy theorem, we have for sufficiently large $n$ that

$$
P\left[Z_{k_{n}} \geq \eta_{n}\right] \geq\left(\frac{1}{2}-\delta\right) \sum_{m=n k_{n}}^{\infty} P\left[\frac{\sum_{j=k_{n}+1}^{m}\left(X_{j}-\mu\right)}{\sqrt{k_{n} \sigma^{2}}}+\frac{\left(m-k_{n}\right) \mu}{\sqrt{k_{n} \sigma^{2}}} \geq \eta_{n}\right] P\left[S_{k_{n}}=m\right] .
$$

But for $m \geq n k_{n}$ we have

$$
\frac{\left(m-k_{n}\right) \mu}{\sqrt{k_{n} \sigma^{2}}} \geq \frac{\left(n k_{n}-k_{n}\right) \mu}{\sqrt{k_{n} \sigma^{2}}}=\eta_{n} .
$$

Hence, for sufficiently large values of $n$,

$$
\begin{aligned}
p\left[S_{k_{n}} \geq \eta_{n}\right] & \geq\left(\frac{1}{2}-\delta\right) \sum_{m=n k_{n}}^{\infty} P\left[\frac{\sum_{j=k_{n}+1}^{m}\left(X_{j}-\mu\right)}{\left.\sqrt{k_{n} \sigma^{2}} \geq 0\right] P\left[S_{k_{n}}=m\right]}\right. \\
& =\left(\frac{1}{2}-\delta\right) \sum_{m=n k_{n}}^{\infty} P\left[\frac{\sum_{j=k_{n}+1}^{m}\left(X_{j}-\mu\right)}{\sqrt{\left(m-k_{n}\right) \sigma^{2}}} \geq 0\right] P\left[S_{k_{n}}=m\right] \\
& \geq\left(\frac{1}{2}-\delta\right)^{2} P\left[S_{k_{n}} \geq n k_{n}\right] \geq\left(\frac{1}{2}-\delta\right)^{2} \varepsilon,
\end{aligned}
$$

i.e., $P\left[Z_{k_{n}} \geq \eta_{n}\right] \geq\left(\frac{1}{2}-\delta\right)^{2} \varepsilon$ for all sufficiently large values of $n$, which violates the assumption that $Z_{n} \stackrel{L^{\prime}}{\longrightarrow} Z$.

The following lemma was proved by B. V. Gnedenko and H. Fahim [2]. 
Lemma 3. Suppose the random variables $\xi_{n 1}, \xi_{n 2}, \ldots$ are identically distributed for every $n$, and suppose there exist a sequence of positive integers $\left\{k_{n}\right\}$ satisfying $k_{n} \uparrow \infty$ and distribution functions $\Phi(x)$ and $A(x)$ such that $P\left[S_{k_{n}}^{(n)} \leq x\right] \rightarrow$ $\Phi(x)$ and $P\left[\frac{\nu_{n}}{k_{n}} \leq x\right] \rightarrow A(x)$ at all $x$ at which $\Phi$ and $A$ are continuous, where $\left\{\nu_{n}\right\}$ are nonnegative integer-valued random variables; $\nu_{n}, \xi_{n 1}, \xi_{n 2}, \ldots$ are independent for each $n$; and $S_{k}^{(n)}=\xi_{n 1}+\cdots+\xi_{n k}$. Then $P\left[S_{\nu_{n}}^{(n)} \leq x\right] \rightarrow \Psi(x)$ at all continuity points of $\Psi(x)$, where $\Psi(x)$ is a distribution function whose characteristic function is $\hat{\Psi}(u)=\int_{0}^{\infty}(\hat{\Phi}(u))^{y} d A(y)$ and $\hat{\Phi}(u)$ is the characteristic function of $\Phi$.

We now use Lemmas 1, 2, and 3 to obtain a stronger conclusion than that of Lemma 1.

Lemma 4. Each condition of the theorem implies that the sequence $\left\{\left(S_{n}-n\right) / \sqrt{n}\right\}$ is tight.

Proof. We need only prove that $Z_{n} \stackrel{\not L}{\longrightarrow} Z$ implies tightness of $\left\{\left(S_{n}-n\right) / \sqrt{n}\right\}$. Let us suppose that this sequence is not tight. Then by Lemma 1 there exists $\varepsilon>0$ and a sequence $k_{n} \uparrow \infty$ such that, say, $P\left[\left(S_{k_{n}}-k_{n}\right) / \sqrt{k_{n}} \geq n\right]>2 \varepsilon$ for all $n$, or

$$
P\left[\frac{S_{k_{n}}-k_{n}}{\sqrt{k_{n} \sigma^{2}}} \mu \geq \frac{n \mu}{\sigma}\right]>2 \varepsilon
$$

for all $n$. By Lemma 2, $\left\{S_{k_{n}} / k_{n}\right\}$ is tight, and hence there exists a subsequence $\left\{m_{n}\right\}$ of $\left\{k_{n}\right\}$ such that $S_{m_{n}} / m_{n} \stackrel{\not}{\longrightarrow}$ (some) $S$ as $n \rightarrow \infty$. Now

$$
Z_{m_{n}}=\frac{1}{\sqrt{m_{n} \sigma^{2}}}\left(X_{1}+\cdots+X_{S_{m_{n}}}-\mu m_{n}\right)=U_{n}+V_{n},
$$

where

$$
U_{n}=\frac{X_{1}+\cdots+X_{S_{m_{n}}}-\mu S_{m_{n}}}{\sqrt{m_{n} \sigma^{2}}}
$$

and

$$
V_{n}=\frac{S_{m_{n}}-m_{n}}{\sqrt{m_{n} \sigma^{2}}} \mu .
$$

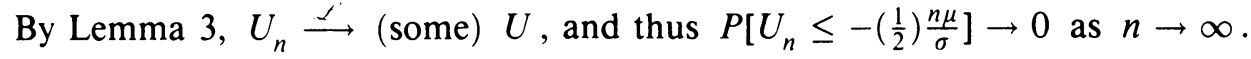
Now

so

$$
\left[Z_{m_{n}}>\frac{n \mu}{2 \sigma}\right] \supset\left[U_{n}>-\frac{n \mu}{2 \sigma}\right]\left[V_{n}>\frac{n \mu}{\sigma}\right] \text {, }
$$

$$
P\left[Z_{m_{n}}>\frac{n \mu}{2 \sigma}\right] \geq P\left[V_{n}>\frac{n \mu}{\sigma}\right]-P\left(\left[U_{n} \leq-\frac{n \mu}{2 \sigma}\right]\left[V_{n}>\frac{n \mu}{\sigma}\right]\right) \text {. }
$$

But

$$
P\left(\left[U_{n} \leq-\frac{n \mu}{2 \sigma}\right]\left[V_{n}>\frac{n \mu}{\sigma}\right]\right) \leq P\left[U_{n} \leq-\frac{n \mu}{2 \sigma}\right] \rightarrow 0 \text { as } n \rightarrow \infty .
$$


Hence for all sufficiently large values of $n$,

$$
P\left[Z_{m_{n}}>\frac{n \mu}{2 \sigma}\right] \geq P\left[V_{n}>\frac{n \mu}{\sigma}\right]-\varepsilon>\varepsilon .
$$

This implies that $\left\{Z_{m_{n}}\right\}$ does not converge in law, which contradicts the hypothesis that $Z_{n} \stackrel{\not}{\longrightarrow} Z$.

Lemma 5. If $a$ and $b$ are real numbers, and if $[b]-[a] \leq-2$, then $b-a<$ -1 .

Proof. We may write $b=[b]+(b)$ and $a=[a]+(a)$, where $0 \leq(b)<1$ and $0 \leq(a)<1$. Then $-1<-(a) \leq 0$, and so $-1<(b)-(a)<1$. Finally, $b-a=[b]-[a]+(b)-(a)<-1$.

Lemma 6. If $K$ is any real number and $f(n)=n+[K \sqrt{n}]$, then for $n$ sufficiently large, $f(n)$ is nondecreasing and $\lim _{n \rightarrow \infty} f(n)=\infty$.

Proof. If $K \geq 0$ the lemma is immediate. Assume $K<0$. Since

$$
\begin{aligned}
\lim _{n \rightarrow \infty}(\sqrt{n+1}-\sqrt{n}) & =\lim _{n \rightarrow \infty}(\sqrt{n+1}-\sqrt{n}) \frac{(\sqrt{n+1}+\sqrt{n})}{(\sqrt{n+1}+\sqrt{n})} \\
& =\lim _{n \rightarrow \infty} \frac{1}{(\sqrt{n+1}+\sqrt{n})}=0,
\end{aligned}
$$

it follows that $K \sqrt{n+1}-K \sqrt{n} \rightarrow 0$. Hence for sufficiently large $n,-\frac{1}{2}<$ $K \sqrt{n+1}-K \sqrt{n} \leq 0$. If $[K \sqrt{n+1}]-[K \sqrt{n}] \leq-2$, then by Lemma 5 $K \sqrt{n+1}-K \sqrt{n}<-1$, which cannot happen for large $n$. Hence $[K \sqrt{n+1}]-$ $[K \sqrt{n}] \geq-1$, or $(n+1)+[K \sqrt{n+1}] \geq n+[K \sqrt{n}]$. Hence $f$ is nondecreasing for large $n$.

Since $-\frac{1}{2}<K \sqrt{n+1}-K \sqrt{n} \leq 0$, it follows that $-1 \leq K \sqrt{n+2}-K \sqrt{n}<0$. Then by Lemma 5 ,

$$
[K \sqrt{n+2}]-[K \sqrt{n}] \geq-1 .
$$

Then $f(n+2)-f(n)=2+[K \sqrt{n+2}]-[K \sqrt{n}] \geq 1, n$ large, and hence $\lim _{n \rightarrow \infty} f(n)=\infty$.

We now prove the theorem. We assume that either condition of the theorem holds. Recalling our notation

$$
Z_{n}=\frac{1}{\sqrt{n \sigma^{2}}}\left(X_{1}+\cdots+X_{S_{n}}-n \mu\right),
$$

we observe that

$$
\begin{aligned}
E\left(e^{i u Z_{n}}\right)=E\left(E\left(e^{i u Z_{n}} \mid S_{n}\right)\right) & =\int_{0}^{\infty} E\left(e^{i u Z_{n}} \mid S_{n}=s\right) d F_{S_{n}}(s) \\
& =\int_{0}^{\infty} E\left(\exp i u \frac{X_{1}+\cdots+X_{s}-n \mu}{\sqrt{n \sigma^{2}}}\right) d F_{S_{n}}(s)
\end{aligned}
$$


Next consider the change of variable $x=(s-n) / \sqrt{n}$ and, correspondingly, $U_{n}=\left(S_{n}-n\right) / \sqrt{n}$, and define

$$
g_{n}(u, x)=E\left(\exp i u \frac{X_{1}+\cdots+X_{n+[x \sqrt{n}]}-(n+[x \sqrt{n}]) \mu}{\sqrt{(n+[x \sqrt{n}]) \sigma^{2}}} C_{n}(x)\right),
$$

where

$$
C_{n}(x)=\sqrt{\frac{n+[x \sqrt{n}]}{n}} \rightarrow 1 \text { as } n \rightarrow \infty \text { uniformly }
$$

over every bounded set. Then

$$
E\left(e^{i u Z n}\right)=\int_{-\infty}^{\infty} g_{n}(u, x)\left(\exp i u \frac{[x \sqrt{n}] \mu}{\sqrt{n \sigma^{2}}}\right) d F_{U_{n}}(x) .
$$

Lemma 7. For each fixed $u \in \mathbf{R}^{1}$ and every bounded interval $[K, L] \subset \mathbf{R}^{1}$, $g_{n}(u, x) \rightarrow e^{-u^{2} / 2}$ as $n \rightarrow \infty$ uniformly in $x \in[K, L]$.

Proof. Let us define $h_{n}(u, x)$ and $\varphi_{n}(u)$ by:

$$
\begin{gathered}
h_{n}(u, x)=E\left(\exp i u \frac{X_{1}+\cdots+X_{n+[x \sqrt{n}]}-\mu(n+[x \sqrt{n}])}{\sqrt{(n+[x \sqrt{n}]) \sigma^{2}}}\right), \\
\varphi_{n}(u)=E\left(\exp i u \frac{X_{1}+\cdots+X_{n}-n \mu}{\sqrt{n \sigma^{2}}}\right) .
\end{gathered}
$$

By the Lindeberg-Lévy theorem, $\varphi_{n}(u) \rightarrow e^{-u^{2} / 2}$ as $n \rightarrow \infty$. Now $u$ is fixed. By Lemma $6, n+[\sqrt{n} K] \uparrow \infty$ as $n \rightarrow \infty$. Let $\varepsilon>0$ be arbitrary. Then there exists a positive integer $n_{0}$ such that $n>n_{0}+\left[\sqrt{n_{0}} K\right]$ implies that

$$
\left|\varphi_{n}(u)-e^{-u^{2} / 2}\right|<\varepsilon .
$$

Let $k(n, x)=n+[x \sqrt{n}]$. By Lemma 6, $k(n+1, x) \geq k(n, x)$ for all $n$ and $x$. Note that $h_{n}(u, x)=\varphi_{k(n, x)}(u)$ and $k(n, x) \geq n+[K \sqrt{n}]$ for all $x \in[K, L]$. Hence for all $n>n_{0}+\left[\sqrt{n_{0}} K\right]$ and all $x \in[K, L],\left|\varphi_{k(n, x)}(u)-e^{-u^{2} / 2}\right|<\varepsilon$. Since $C_{n}(x) \rightarrow 1$ as $n \rightarrow \infty$ uniformly in $x \in[K, L]$, then for any $\eta>0$ there exists a positive integer $n_{1}$ such that $n>n_{1}$ implies $\left|C_{n}(x)-1\right|<\eta$ for all $x \in[K, L]$. We observe that

$$
\begin{aligned}
\left|g_{n}(u, x)-e^{-u^{2} / 2}\right| & =\left|\varphi_{k(n, x)}\left(C_{n}(x) u\right)-e^{-u^{2} / 2}\right| \\
& \leq\left|\varphi_{k(n, x)}\left(C_{n}(x) u\right)-\varphi_{k(n, x)}(u)\right|+\left|\varphi_{k(n, x)}(u)-e^{-u^{2} / 2}\right| .
\end{aligned}
$$

Because of the fact that $\left\{\varphi_{1}(t), \varphi_{2}(t), \ldots, e^{-t^{2} / 2}\right\}$ are uniformly equicontinuous (see Theorem 2 on page 94 of [6]), there exists $\eta>0$ such that

$$
\left|\varphi_{n}\left(t^{\prime}\right)-\varphi_{n}\left(t^{\prime \prime}\right)\right|<\varepsilon \text { for all } t^{\prime}, t^{\prime \prime}
$$


which satisfy $\left|t^{\prime}-t^{\prime \prime}\right|<\eta$. Hence for all $n>\max \left\{n_{1}, n_{0}+\left[\sqrt{n_{0}} K\right]\right\}$ we have

$$
\left|\varphi_{k(n, x)}\left(C_{n}(x) u\right)-\varphi_{k(n, x)}(u)\right|<\varepsilon \quad \text { for all } x \in[K, L] .
$$

Thus for fixed $u$ and all sufficiently large values of $n$,

$$
\left|\varphi_{k(n, x)}\left(C_{n}(x) u\right)-e^{-u^{2} / 2}\right|<2 \varepsilon \quad \text { for all } x \in[K, L] \text {. }
$$

Let us define $r_{n}(x)$ by

$$
r_{n}(x)=\exp i u \frac{[x \sqrt{n}] \mu}{\sqrt{n \sigma^{2}}}
$$

we note that $\left|r_{n}(x)\right|=1$. By Lemma 4, given an arbitrary $\varepsilon>0$ there exists $K_{\varepsilon}>0$ such that $F_{U_{n}}\left(-K_{\varepsilon}\right)<\varepsilon$ and $1-F_{U_{n}}\left(K_{\varepsilon}\right)<\varepsilon$ for all $n$. We may write

$$
\begin{aligned}
E\left(e^{i u Z_{n}}\right)= & \int_{-\infty}^{\infty}\left(g_{n}(u, x)-e^{-u^{2} / 2}\right) r_{n}(x) d F_{U_{n}}(x) \\
& +e^{-u^{2} / 2} \int_{-\infty}^{\infty} r_{n}(x) d F_{U_{n}}(x) \\
= & \int_{-K_{\varepsilon}}^{K_{\varepsilon}}\left(g_{n}(u, x)-e^{-u^{2} / 2}\right) r_{n}(x) d F_{U_{n}}(x)+Q_{n}(u, \varepsilon) \\
& +e^{-u^{2} / 2} \int_{-\infty}^{\infty} r_{n}(x) d F_{U_{n}}(x),
\end{aligned}
$$

where $\left|Q_{n}(u, \varepsilon)\right| \leq 4 \varepsilon$ for all $u$. Now, whichever condition of the theorem is true, we have by Lemma 7 that

$$
\begin{aligned}
& \left|\int_{-K_{\varepsilon}}^{K_{\varepsilon}}\left(g_{n}(u, x)-e^{-u^{2} / 2}\right) r_{n}(x) d F_{U_{n}}(x)\right| \\
& \quad \leq \sup \left\{\left|g_{n}(u, x)-e^{-u^{2} / 2}\right|:-K_{\varepsilon} \leq x \leq K_{\varepsilon}\right\} \rightarrow 0
\end{aligned}
$$

as $n \rightarrow \infty$.

First assume that $Z_{n} \stackrel{L^{\prime}}{\longrightarrow}$ (some) $Z$ as $n \rightarrow \infty$. Then these last established inequalities imply that for sufficiently large values of $n$,

$$
\limsup _{n \rightarrow \infty}\left|E\left(e^{i \cdot Z_{n}}\right)-e^{-u^{2} / 2} \int_{-\infty}^{\infty} r_{n}(x) d F_{U_{n}}(x)\right| \leq 4 \varepsilon .
$$

Thus

$$
e^{-u^{2} / 2} \int_{-\infty}^{\infty} r_{n}(x) d F_{U_{n}}(x) \rightarrow E\left(e^{i u Z}\right) \text { as } n \rightarrow \infty,
$$

from which we obtain that $\left\{\int_{-\infty}^{\infty} r_{n}(x) d F_{U_{n}}(x)\right\}$ converges to a function of $u$ which is continuous and equals 1 at $u=0$. By the Lévy continuity theorem, there exists a random variable $U$ such that

$$
\frac{\left[U_{n} \sqrt{n}\right] \mu}{\sqrt{n \sigma^{2}}} \stackrel{t^{\prime}}{\longrightarrow} \frac{U \mu}{\sigma} \text {. }
$$


Since

$$
\frac{\left[U_{n} \sqrt{n}\right] \mu}{\sqrt{n \sigma^{2}}}=\frac{\left(\left[U_{n} \sqrt{n}\right]-U_{n} \sqrt{n}\right) \mu}{\sqrt{n \sigma^{2}}}+\frac{U_{n} \mu}{\sigma},
$$

and since

$$
\left|\frac{\left(\left[U_{n} \sqrt{n}\right]-U_{n} \sqrt{n}\right) \mu}{\sqrt{n \sigma^{2}}}\right| \leq \frac{\mu}{\sqrt{n \sigma^{2}}} \rightarrow 0 \quad \text { as } n \rightarrow \infty,
$$

it follows that

$$
\frac{\left(\left[U_{n} \sqrt{n}\right]-U_{n} \sqrt{n}\right) \mu}{\sqrt{n \sigma^{2}}} \stackrel{P}{\longrightarrow} 0 .
$$

Thus by the well-used result of Cramér ([1], page 254) it follows that $U_{n} \mu / \sigma \stackrel{\iota^{\prime}}{\longrightarrow}$ $U \mu / \sigma$ as $n \rightarrow \infty$; i.e., $\left(S_{n}-n\right) / \sqrt{n} \stackrel{\mathscr{L}}{\longrightarrow} U$ as $n \rightarrow \infty$, and the distribution of $Z$ is that of $X+Y$, where $X$ and $Y$ are independent, $X$ being $\mathcal{N}(0,1)$ and $Y$ having the same distribution as $U \mu / \sigma$.

Conversely, suppose that $\left(S_{n}-n\right) / \sqrt{n} \stackrel{\mathscr{L}}{\longrightarrow} U$ as $n \rightarrow \infty$ for some random variable $U$, i.e., $U_{n} \stackrel{\mathscr{L}^{\prime}}{\longrightarrow} U$. We first observe that

$$
\begin{aligned}
\left|\exp i u \frac{[x \sqrt{n}] \mu}{\sqrt{n \sigma^{2}}}-\exp i u \frac{x \mu}{\sigma}\right| \\
=\left|\left(\exp i u \frac{x \mu}{\sigma}\right)\left(\left(\exp i u\left(\frac{[x \sqrt{n}] \mu}{\sqrt{n \sigma^{2}}}-\frac{x \mu}{\sigma}\right)\right)-1\right)\right| .
\end{aligned}
$$

But

$$
\left|\frac{[x \sqrt{n}] \mu}{\sqrt{n \sigma^{2}}}-\frac{x \mu}{\sigma}\right| \leq \frac{\mu}{\sqrt{n} \sigma} \rightarrow 0
$$

uniformly in $x$ as $n \rightarrow \infty$, which from the previous inequality implies for each fixed $u$ that

$$
\left|\exp i u \frac{[x \sqrt{n}] \mu}{\sqrt{n \sigma^{2}}}-\exp i u \frac{x \mu}{\sigma}\right| \rightarrow 0 \text { as } n \rightarrow \infty
$$

uniformly in $x$. Hence for fixed $u$,

$$
\begin{aligned}
\left|E\left(e^{i u Z_{n}}\right)-e^{-u^{2} / 2} E\left(e^{\left.i u \mu U^{\prime} / \sigma\right)}\right)\right| & \leq \int_{-\infty}^{\infty}\left|g_{n}(u, x)-e^{-u^{2} / 2}\right|\left|r_{n}(x)\right| d F_{U_{n}}(x) \\
& +\int_{-\infty}^{\infty} e^{-u^{2} / 2}\left|\exp i u \frac{[x \sqrt{n}] \mu}{\sqrt{n \sigma^{2}}}-e^{i u \mu x / \sigma}\right| d F_{U_{n}}(x) \\
& +\left|\int_{-\infty}^{\infty} e^{i u \mu x / \sigma} d F_{U_{n}}(x)-\int_{-\infty}^{\infty} e^{i u \mu x / \sigma} d F_{U}(x)\right| .
\end{aligned}
$$

Let us denote the three summands on the right side of the above inequality by $\alpha_{n}, \beta_{n}$, and $\gamma_{n}$ respectively. The hypothesis $U_{n} \stackrel{t^{\prime}}{\longrightarrow} U$ implies that $\gamma_{n} \rightarrow 0$ as $n \rightarrow \infty$ by the continuity theorem. Since $\left\{U_{n}\right\}$ is tight, given any $\varepsilon>0$ 
there exists $K_{\varepsilon}>0$ such that $F_{U_{n}}\left(-K_{\varepsilon}\right)<\varepsilon$ and $1-F_{U_{n}}\left(K_{\varepsilon}\right)<\varepsilon$ for all $n$. Since $\left|g_{n}(u, x)\right| \leq 1$ for all $n, u, x$, it follows that

$$
\alpha_{n} \leq 4 \varepsilon+\int_{-K_{\varepsilon}}^{K_{\varepsilon}}\left|g_{n}(u, x)-e^{-u^{2} / 2}\right| d F_{U_{n}}(x),
$$

and thus by Lemma 7 ,

$$
\alpha_{n} \leq 4 \varepsilon+\sup _{|x| \leq K_{\varepsilon}}\left|g_{n}(u, x)-e^{-u^{2} / 2}\right|<5 \varepsilon
$$

for all large values of $n$. Finally,

$$
\beta_{n} \leq \sup _{x}\left|\exp i u \frac{[x \sqrt{n}] \mu}{\sqrt{n \sigma^{2}}}-\exp i u \frac{\mu x}{\sigma}\right| \rightarrow 0
$$

as $n \rightarrow \infty$ as previously established. This proves that $Z_{n} \stackrel{\mathscr{L}}{\longrightarrow} Z$ as $n \rightarrow \infty$ and concludes the proof of the theorem.

\section{REFERENCES}

1. H. Cramér, Mathematical methods of statistics, Princeton Univ. Press, Princeton, New Jersey, 1946.

2. B. V. Gnedenko and H. Fahim, On a transfer theorem, Soviet Math. Dokl. 10 (1969), 769-772.

3. Z. Rychlik, A central limit theorem for sums of a random number of independent random variables, Colloq. Math. 35 (1976), 147-158.

4. A. Rényi, On the central limit theorem for the sum of a random number of independent random variables, Acta Math. Hungar. 11 (1960), 97-102.

5. J. G. Shanthikumar and U. Sumita, A central limit theorem for random sums of random variables, Oper. Res. Lett. 3 (1984), 153-155.

6. H. G. Tucker, A graduate course in probability, Academic Press, New York, 1967.

Mathematics Department, University of California at Irvine, Irvine, California 92717 